\title{
Production of Hypernuclei by Antiprotons
}

\author{
Horst Lenske ${ }^{1, \star}$ and Alexei Larionov ${ }^{1,2, \star \star}$ \\ ${ }^{1}$ Institut für Theoretische Physik, Justus-Liebig-Universität Gießen, Germany \\ ${ }^{2}$ Frankfurt Institute for Advanced Studies (FIAS), D-60438 Frankfurt am Main, Germany
}

\begin{abstract}
The production of hypernuclei is investigated for $\bar{p}+A \rightarrow \bar{\Lambda}+{ }_{\Lambda} A$ reactions in a covariant meson exchange approach. Besides the conventional pseudo-scalar $(K)$ and vector $\left(K^{*}\right)$ channels, we study for the first time also contributions from the correlated $\pi K$ scalar channel, described by the $\kappa / K_{0}^{*}$ meson. Initial and final state interactions are considered by eikonal theory. The total and angular differential cross sections of the coherent process $\bar{p}+{ }^{A} Z \rightarrow{ }_{\Lambda}^{A}(Z-1)+\bar{\Lambda}$ are evaluated at the beam momenta $1.5 \ldots 20 \mathrm{GeV} / \mathrm{c}$ within the meson exchange model with bound proton and $\Lambda$-hyperon wave functions. It is shown that the shape of the beam momentum dependence of the hypernucleus production cross sections with various discrete $\Lambda$ states is strongly sensitive to the presence of the scalar $\kappa$ meson exchange in the $\bar{p} p \rightarrow \bar{\Lambda} \Lambda$ amplitude. This can be used as a clean test of the exchange by scalar $\pi K$ correlation in coherent $\bar{p} A$ reactions.
\end{abstract}

\section{Introduction}

Hypernuclei are being produced in manifold ways, by photon-, pion-, antikaon-, proton-, antiprotonand nucleus-nucleus interactions [1]. All these types of reactions, except the $\bar{p} A$ one, are rather well studied both experimentally and theoretically. But up to now, there are only few theoretical studies of hypernuclear production in antiproton-nucleus interactions [2-5], and all of them address the incoherent production mechanism. Incoherent hypernucleus production in central collisions is initiated by antikaon production in elementary in-medium $\bar{p} N$ annihilation reactions followed by a sequence of strangeness exchange reactions of the type $\bar{K} N \rightarrow Y \pi$. Such reactions are the ideal tool to investigate simultaneously the production of single- and multi-strangeness systems, as discussed e.g. in our recent review article [6]. However, a different approach is required if spectroscopic studies of the final hypernuclei are the aim. For that purpose, the proper method are peripheral reactions by which hypernuclei in bound discrete quantum states are obtained. A comprehensive overview of the status of such studies is found in the recent reviews in [7] and [6]. We have investigated previously coherent hypernuclear production for proton- and pion-induced reactions in [8] and [9], respectively, and for photo-production in [10]. Since such coherent reactions are of a perturbative character, they can be described quantum mechanically by distorted wave methods.

Not much is known, in fact, on the basic $\bar{p}+p \rightarrow \bar{\Lambda}+\Lambda$ reaction amplitude. Here, we use a meson exchange model. On the antibaryon side a $\bar{u}$-quark must be changed into a $\bar{s}$ quark while on the baryon side a $u$-quark has to be transformed into a $s$-quark. That can be viewed as the propagation of

\footnotetext{
^e-mail: horst.lenske@physik.uni-giessen.de

$\star \star$ e-mail: larionov@fias.uni-frankfurt.de
} 
positively charged mesons of a $[u \bar{s}]$ quark structure with strangeness $|S|=1$ from baryon to antibaryon (or of $[\bar{u} s]$ mesons with strangeness $S=-1$ in the opposite direction). Obvious candidates for such a process are the pseudo-scalar $\left(0^{-}\right)$kaon $K$ and vector $\left(1^{-}\right) K^{*}$ mesons. However, we have to expect that also $\pi K$ correlated exchange in the scalar $0^{+}$-channel may play an important role. The $0^{+},|S|=1$ channel is represented by the $K_{0}^{*}(800)$ or $\kappa$ mesons [11] which may be considered as the $|S|=1$ members of the (hypothetical) scalar meson octet to which also the $\sigma / f_{0}(600)$ and the $\delta / a_{0}(980)$ mesons belong. As for the other $0^{+}$-mesons also the $\kappa$ meson is characterized by a rather broad spectral distribution with uncertain mass and width. In the present context, the $\kappa / K_{0}^{*}$ meson contributes of course through $t$-channel exchange processes. In that sense, we consider $\kappa$ exchange as an economical way to account for the correlated $\pi K$ channel. The $\kappa$ exchange channel is of particular interest for multi-strangeness baryonic matter as found in heavy ion collisions and in neutron stars. $\kappa$ exchange is also an indispensable part of baryon-baryon interaction approaches utilizing the $S U(3)$-flavour group structure. The Nijmegen group was probably the first one to introduce that channel explicitly $[12,13]$ into their treatment of baryon-baryon scattering while in the Juelich model that channel is treated dynamically as a $\pi K$-correlation [14]. We note that in the present context the unnatural parity $K$-exchange is strongly suppressed for transition involving bound proton and $\Lambda$ states, respectively, because it is a purely relativistic effect proceeding through the lower wave function components of the Dirac spinors. Thus, coherent hypernucleus production reactions are perfect tools to addressing specifically the exchange of the natural parity $K^{*}$ and $\kappa$ mesons. Any other independent source of information allowing to probe hyperon-nucleon and hyperon-nucleus interactions is highly wanted. In this respect, hypernuclear reaction physics may provide important clues.

In section 2 we introduce the Lagrangian describing our covariant production model and the relativistic mean-field approach for bound baryon states. Initial and final state interactions of the scattered antibaryons are taken into account in the eikonal approximation. In section 3 we study the population of discrete bound states in the ${ }_{\Lambda}^{40} \mathrm{Cl}$ hypernucleus. Angular distributions and total hypernucleus production cross sections are discussed. In section 4 we summarize our results and present our conclusions.

\section{Theory of Coherent Antiproton-Nucleus Reactions}

Investigations of peripheral reactions with strongly absorbed hadrons require a reliable model for elastic scattering in order to account properly for the extremely important initial (ISI) and final state (FSI) interactions. ISI and FSI will affect both the magnitude and the shape of momentum and angular distributions. Thus, descriptions using lowest order Born approximations as the plane wave approach underlying the Impulse Approximation (IA) do not lead to reliable results. In [15], a Glauber approach was developed for elastic scattering of antiprotons on nuclei, including also investigations of spin-observables. Data of total cross sections as well as angular distributions of cross sections and azimuthal asymmetries and polarization observables are well described over large ranges of energies, corresponding to $p_{\text {lab }}$ from a few hundred $\mathrm{MeV} / \mathrm{c}$ to $20 \mathrm{GeV} / \mathrm{c}$, and over a wide region of momentum transfers. Typical results for elastic scattering angular distributions are displayed in Fig. 1, showing that the LEAR data are well reproduced. A detailed discussion of the dependencies of elastic scattering cross sections and polarization variables on incident energy and target mass and the comparison to proton elastic scattering are found in [15]. For the present case of high energy studies, eikonal theory is used.

The basic $\bar{p}+p \rightarrow \bar{\Lambda}+\Lambda$ reaction amplitude is not well explored. Here, we use a meson exchange model. The exchange of $K, K^{*}$ and $\kappa$ mesons, respectively, is introduced by the following interaction 

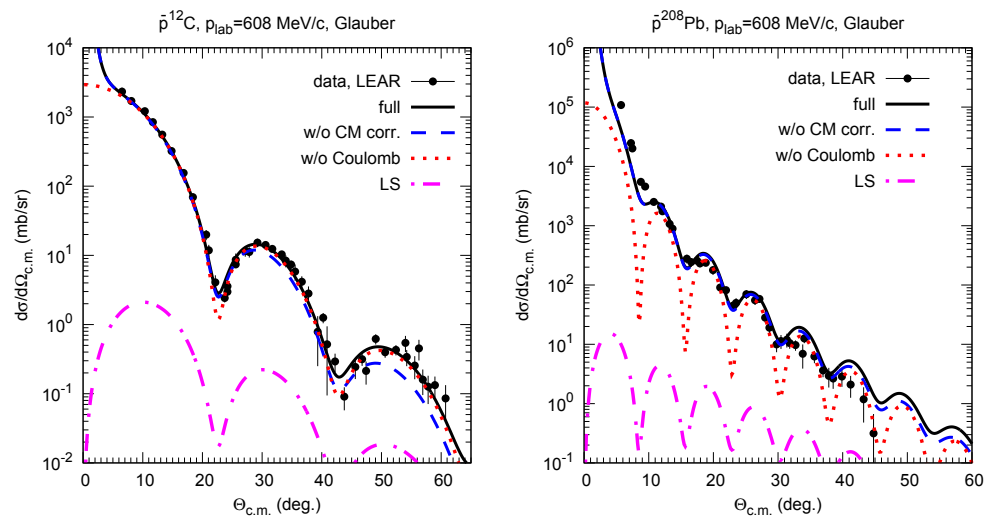

Figure 1. Angular differential cross section of proton elastic scattering on ${ }^{12} \mathrm{C}$ and ${ }^{208} \mathrm{~Pb}$ and beam momentum $p_{\text {lab }}=608 \mathrm{MeV} / \mathrm{c}$ corresponding to $T_{\text {lab }}=180 \mathrm{MeV}$. Contributions of various interaction are shown. For more details see Ref. [15]. The data are from ref. [16].

Lagrangians [17-19]:

$$
\begin{aligned}
\mathcal{L}_{K N \Lambda} & =-i g_{K N \Lambda} \bar{N} \gamma^{5} \Lambda K+\text { h.c. } \\
\mathcal{L}_{K^{*} N \Lambda} & =\bar{N}\left(G_{v} \gamma^{\mu}-\frac{G_{t} \sigma^{\mu v} \partial_{v}^{K^{*}}}{m_{N}+m_{\Lambda}}\right) \Lambda K_{\mu}^{*}+\text { h.c. }, \\
\mathcal{L}_{\kappa N \Lambda} & =-g_{\kappa N \Lambda} \bar{N} \Lambda \kappa+\text { h.c. }
\end{aligned}
$$

In the case of the bound proton and $\Lambda$ we include their wave functions in the field operators of the Lagrangians Eqs. (1)-(3) and calculate the $S$-matrix in the second order perturbation theory, as discussed in [20]. The coupling constants will be discussed below in section 2.1

The $K, K^{*}$ and $\kappa$ exchange (noninvariant) partial matrix elements are expressed as

$$
\begin{aligned}
& \mathcal{M}_{K}=-g_{K N \Lambda}^{2} F_{K}^{2}(t) \sqrt{\Omega} \bar{u}_{-p_{1},-\lambda_{1}} \gamma^{5} u_{-p_{3},-\lambda_{3}} \frac{1}{t-m_{K}^{2}} \int d^{3} r e^{-i q \boldsymbol{r}} \bar{\psi}_{4}(\boldsymbol{r}) \gamma^{5} \psi_{2}(\boldsymbol{r}), \\
& \mathcal{M}_{K^{*}}=-F_{K^{*}}^{2}(t) \sqrt{\Omega} \bar{u}_{-p_{1},-\lambda_{1}} \Gamma^{\mu}(-q) u_{-p_{3},-\lambda_{3}} G_{\mu \nu}(q) \int d^{3} r e^{-i q r} \bar{\psi}_{4}(\boldsymbol{r}) \Gamma^{\nu}(q) \psi_{2}(\boldsymbol{r}), \\
& \mathcal{M}_{\kappa}=g_{\kappa N \Lambda}^{2} F_{\kappa}^{2}(t) \sqrt{\Omega} \bar{u}_{-p_{1},-\lambda_{1}} u_{-p_{3},-\lambda_{3}} \frac{1}{t-m_{\kappa}^{2}} \int d^{3} r e^{-i \boldsymbol{q} \boldsymbol{r}} \bar{\psi}_{4}(\boldsymbol{r}) \psi_{2}(\boldsymbol{r}) .
\end{aligned}
$$

Here, $\psi_{2}(\boldsymbol{r})$ and $\psi_{4}(\boldsymbol{r})$ are the relativistic wave functions of the bound proton and $\Lambda$, respectively.

$p_{i}$ is the four-momentum and $\lambda_{i}= \pm 1 / 2$ is the spin magnetic quantum number of an antiproton $(i=1)$ and anti-Lambda $(i=3) . q=p_{3}-p_{1}$ is the four-momentum transfer, $t=q^{2}$. In Eq. (5),

$$
G_{\mu \nu}(q)=\frac{-g_{\mu \nu}+q_{\mu} q_{\nu} / m_{K^{*}}^{2}}{t-m_{K^{*}}^{2}}
$$

is the $K^{*}$ meson propagator. We neglected the widths of the $K^{*}$ and $\kappa$ mesons in their propagators as the momentum transfers are space-like (e.g. $-t=0.08 \ldots 1.7 \mathrm{GeV}^{2}$ at $p_{\text {lab }}=2 \mathrm{GeV} / \mathrm{c}$ ). The $K^{*} N \Lambda$ vertex function is defined as

$$
\Gamma^{\mu}(q)=i G_{v} \gamma^{\mu}+\frac{G_{t}}{m_{N}+m_{\Lambda}} \sigma^{\mu v} q_{v}
$$


Monopole vertex form factors $F_{K, K^{*}, K}$ are used:

$$
F_{j}(t)=\frac{\Lambda_{j}^{2}-m_{j}^{2}}{\Lambda_{j}^{2}-t}, \quad j=K, K^{*}, \kappa
$$

Similar to refs. [21, 22] we included in Eqs. (4)-(6) the attenuation factor $\sqrt{\Omega}$ to describe the modification of the elementary $\bar{p} p \rightarrow \bar{\Lambda} \Lambda$ amplitude due to ISI in the $\bar{p} p$ channel and FSI in the $\bar{\Lambda} \Lambda$ channel, which corresponds to the absorptive potentials.

The differential cross section in the rest frame of the target nucleus is written as

$$
d \sigma=\frac{2 \pi \delta^{(4)}\left(p_{1}+p_{A}-p_{3}-p_{B}\right)}{2 p_{\text {lab }}} \frac{1}{2} \sum_{\lambda_{1}, m_{2}, \lambda_{3}, m_{4}}\left|\mathcal{M}_{K}+\mathcal{M}_{K^{*}}+\mathcal{M}_{\kappa}\right|^{2} \frac{d^{3} p_{3}}{(2 \pi)^{3} 2 E_{3}} d^{3} p_{B},
$$

where $p_{A}$ and $p_{B}$ are the four momenta of the initial nucleus $(A)$ and final hypernucleus $(B)$. The $\delta$ function in Eq. (10) takes into account the recoil of the hypernucleus. $m_{2}$ and $m_{4}$ are the total angular momentum magnetic quantum numbers of the occupied proton state from the valence shell and of the $\Lambda$ hyperon, respectively. The factor of $1 / 2$ expresses the averaging over $\lambda_{1}$.

\subsection{Coupling Constants}

The choice of coupling constants is based on $S U(3)$ relations [23]:

$$
\begin{aligned}
g_{K N \Lambda} & =-g_{\pi N N} \frac{3-2 \alpha_{P S}}{\sqrt{3}}, \\
G_{v, t} & =-G_{v, t}^{\rho} \frac{3-2 \alpha_{E, M}}{\sqrt{3}}, \\
g_{\kappa N \Lambda} & =-g_{\sigma N N} \frac{3-2 \alpha_{S}}{3-4 \alpha_{S}},
\end{aligned}
$$

where $\alpha$ 's are the $D$-type coupling ratios. The $\pi N N$ coupling constant is very well known, $g_{\pi N N}=13.4$ [24]. The vector $\rho N N$ coupling constant is also fixed, $G_{v}^{\rho}=2.66$, however, the tensor $\rho N N$ coupling constant is quite uncertain, $G_{t}^{\rho}=10.9 \ldots 20.6$ [17]. The $\sigma N N$ coupling constant can be estimated either from the Bonn model [25] or from the Walecka-type models (cf. [26]). In both cases one obtains $g_{\sigma N N} \simeq 10$. The $\alpha$ 's for the octets of light pseudoscalar and vector mesons are reasonably well determined [17, 19]: $\alpha_{P S} \simeq 0.6, \alpha_{E} \simeq 0, \alpha_{M} \simeq 3 / 4$. However, there is no any phenomenological information on $\alpha_{S}$.

Thus, the coupling constants $G_{t}$ and $g_{\kappa N \Lambda}$, the cutoff parameters $\Lambda_{K}, \Lambda_{K^{*}}$ and $\Lambda_{\kappa}$, and the attenuation factor $\Omega$ remain to be determined from comparison with experimental data. We adjusted these parameters to describe the beam momentum dependence of the $\bar{p} p \rightarrow \bar{\Lambda} \Lambda$ cross section. The two sets of parameters, (1) without $\kappa$ meson and (2) with $\kappa$ meson, are listed in Table 1 . In the calculations we used the mass $m_{\kappa}=682 \mathrm{MeV}$ [27].

As we see from Fig. 2 , in the calculation with set 1 the peak of the $\bar{p} p \rightarrow \bar{\Lambda} \Lambda$ cross section at $p_{\text {lab }} \simeq 2 \mathrm{GeV} / \mathrm{c}$ is exhausted by the $K$ exchange. In contrast, in the case of set 2 the peak is exhausted mostly by the $\kappa$ exchange. The $K^{*}$ exchange contribution grows with beam momentum and becomes dominant at $p_{\text {lab }}>3 \mathrm{GeV} / \mathrm{c}$. We note that set 2 gives a steeper increasing angular differential cross section towards $\Theta_{\text {c.m. }}=0$ at $p_{\text {lab }}=2.060 \mathrm{GeV} / \mathrm{c}$ in a good agreement with experimental data [30]. 
Table 1. Parameters of the $\bar{p} p \rightarrow \bar{\Lambda} \Lambda$ amplitude. The value of $g_{K N \Lambda}$ slightly differs from -13.3 as given by Eq. (11) and is taken from from $K^{+} N$ scattering analysis of ref. [28]. The cutoff parameters $\Lambda_{K}, \Lambda_{K^{*}}$ and $\Lambda_{K}$ are in $\mathrm{GeV}$. The attenuation factors are shown in the last column.

\begin{tabular}{|c|l|l|l|l|c|c|c|c|}
\hline Set & $g_{K N \Lambda}$ & $G_{v}$ & $G_{t}$ & $g_{K N \Lambda}$ & $\Lambda_{K}$ & $\Lambda_{K^{*}}$ & $\Lambda_{K}$ & $\Omega$ \\
\hline 1 & -13.981 & -4.6 & -8.5 & - & 2.0 & 1.6 & - & 0.0150 \\
2 & -13.981 & -4.6 & -9.0 & -7.5 & 1.8 & 2.0 & 1.8 & 0.0044 \\
\hline
\end{tabular}
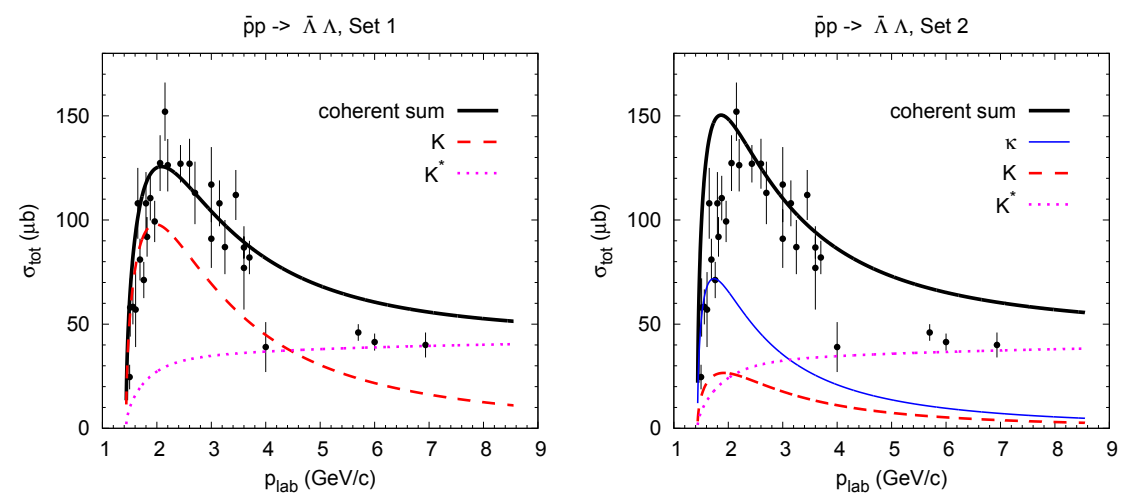

Figure 2. Angle-integrated cross section of the elementary process $\bar{p} p \rightarrow \bar{\Lambda} \Lambda$ as a function of the beam momentum calculated without (Set 1) and with (Set 2) inclusion of the $\kappa$ meson. Experimental data are from ref. [29].

Table 2. Binding energies of the $\Lambda$ states in the ${ }_{\Lambda}^{40} \mathrm{Cl}$ nucleus. Empirical $\Lambda$ binding energies (spin-orbit splitting not resolved) for ${ }_{\Lambda}^{40} \mathrm{Ca}$ from ref. [31] are given in brackets.

\begin{tabular}{|l|l|}
\hline$\Lambda$ state & $B_{\Lambda}[\mathrm{MeV}]$ \\
\hline $1 s_{1 / 2}$ & $18.55(18.7 \pm 1.1)$ \\
$1 p_{3 / 2}$ & $10.20(9.9 \pm 1.1)$ \\
$1 p_{1 / 2}$ & $9.26(9.9 \pm 1.1)$ \\
$1 d_{5 / 2}$ & $2.14(1.5 \pm 1.1)$ \\
$2 s_{1 / 2}$ & 1.44 \\
$1 d_{3 / 2}$ & $0.84(1.5 \pm 1.1)$ \\
\hline
\end{tabular}

\subsection{Nucleon and Hyperon States}

The nucleon and hyperon $(B=N, \Lambda)$ single particle bound state wave functions are determined as solutions of a static Dirac equation with scalar and vector potentials (cf. refs. [9, 31, 32]):

$$
\left(-i \boldsymbol{\alpha} \cdot \boldsymbol{\nabla}+\beta m_{B}^{*}(r)+V_{B}(r)+V_{C}(r)-\varepsilon\right) \psi_{B}(\boldsymbol{r})=0,
$$

where $m_{B}^{*}(r)=m_{B}+S_{B}(r)$ is the effective (Dirac) mass. Both the scalar $\left(S_{B}\right)$ and nuclear vector $\left(V_{B}\right)$ potentials are chosen in the form of superpositions of the classical meson fields, $\sigma\left(I=0, J^{P}=\right.$ 

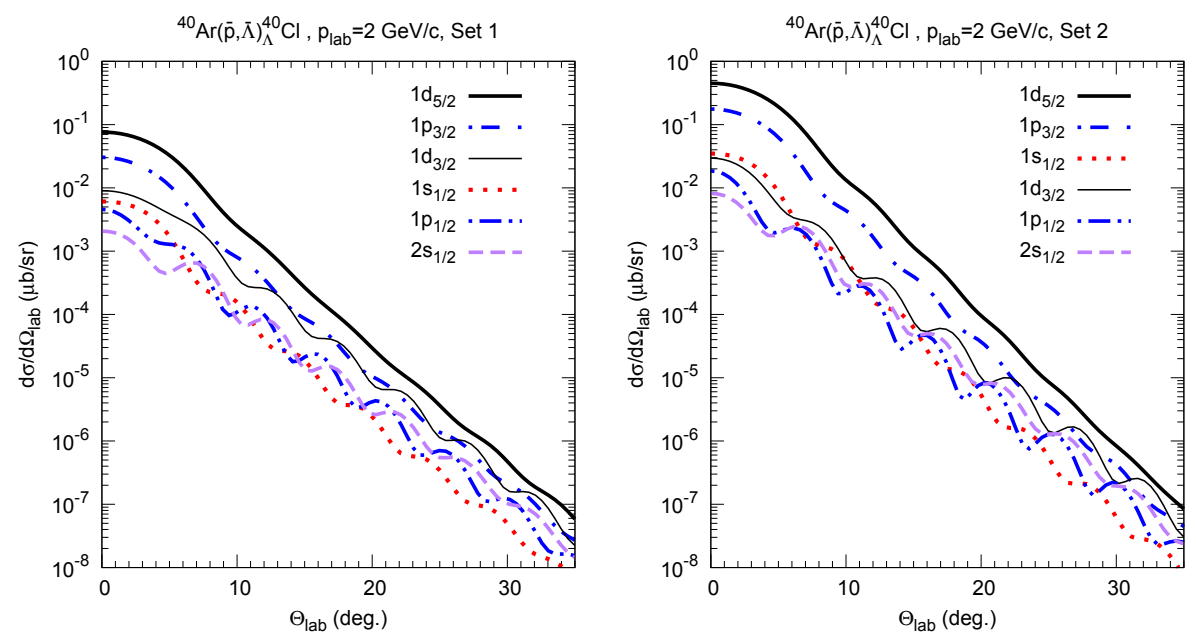

Figure 3. Angular differential cross section of the reaction ${ }^{40} \operatorname{Ar}(\bar{p}, \bar{\Lambda}){ }_{\Lambda}^{40} \mathrm{Cl}$ at $p_{\text {lab }}=2 \mathrm{GeV} / \mathrm{c}$. Lines show the calculations for $\Lambda$ in various states, as indicated. Left and right panels display calculations without (Set 1$)$ and with (Set 2) $\kappa$ exchange.

$\left.0^{+}\right), \omega\left(0,1^{-}\right), \delta\left(1,0^{+}\right)$and $\rho\left(1,1^{-}\right)$, weighted by the strong interaction coupling constants appropriate for the given baryon. For the present purpose, the meson fields are parameterized by Woods-Saxon form factors. For protons also the static Coulomb potential $\left(V_{C}\right)$ contributes [31]. The obtained $\Lambda$ energy levels are in good agreement with the empirical systematics and with our previous relativistic mean-field calculations [31], as seen from Table 2. A detailed discussion of the model parameters and spectroscopic results for $\Lambda$ binding energies and wave functions is found in [20].

\section{Results on Hypernuclear Production by Peripheral Antiproton Reactions}

An appropriate case for exploratory studies is ${ }^{40} \mathrm{Ar}$ which may be of potential interest as target material for future experiments at PANDA@FAIR. In order to assure that after the reaction the residual core nucleus carries as little excitation energy as possible, we consider only strangeness creation processes on protons in the $1 d_{3 / 2}$ valence shell of ${ }^{40} \mathrm{Ar}$.

The differential hypernuclear production cross sections with the $\Lambda$ occupying various shells are compared in Fig. 3. Irrespective of spin-orbit effects, overall the cross sections are larger for larger hyperon orbital angular momentum, i.e. $l_{\Lambda}$. This is a consequence of the interplay of several effects:

- The momentum transfer at $\Theta=0$ is small $(\sim 0.3 \mathrm{GeV} / \mathrm{c})$ implying a suppression of the $p \rightarrow \Lambda$ transitions with large orbital momentum transfer.

- The number of the spin states of the $\Lambda$ contributing to the transition probability, i.e. $2\left(2 l_{\Lambda}+1\right)$, grows obviously with $l_{\Lambda}$.

- For $\Lambda$ states with larger $l_{\Lambda}$ the hyperon probability distribution is increasingly shifted to larger radii. Hence, the absorption effects are diminished with increasing $l_{\Lambda}$.

The inclusion of $\kappa$ exchange leads to significant enhancement of the cross sections at small polar angles for all states of the produced hypernucleus. 

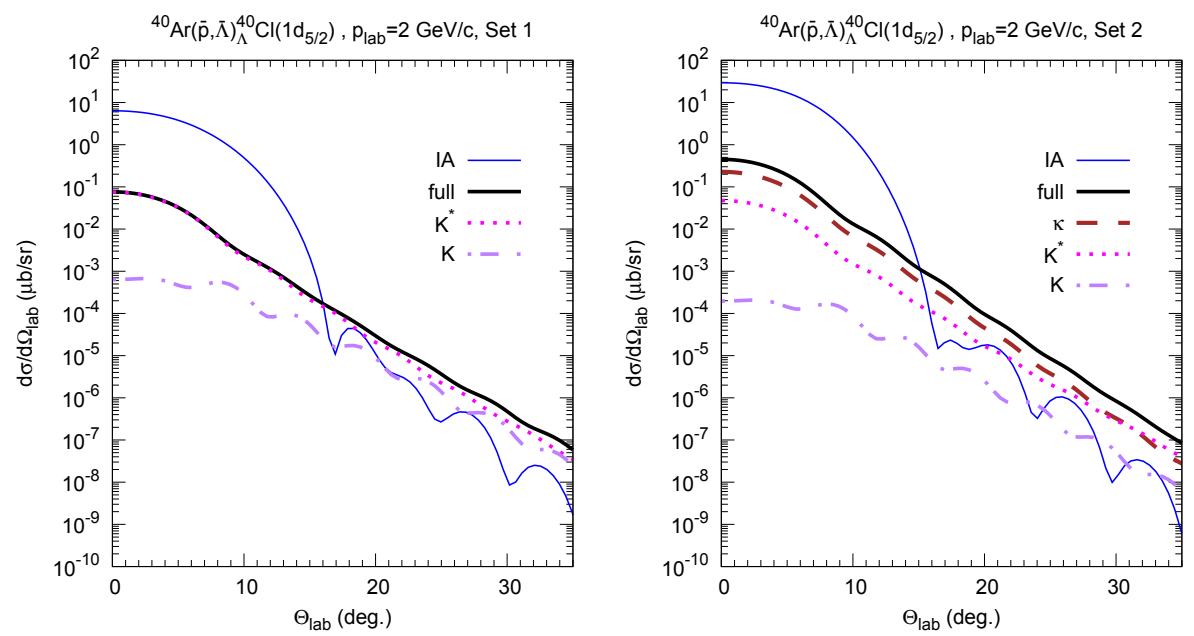

Figure 4. Angular differential cross section of the reaction ${ }^{40} \operatorname{Ar}(\bar{p}, \bar{\Lambda}){ }_{\Lambda}^{40} \mathrm{Cl}$ at $p_{\text {lab }}=2 \mathrm{GeV} / \mathrm{c}$ with $1 d_{5 / 2} \Lambda$ state. As indicated, the IA calculation, the full calculation (with absorption), and the separate meson contributions to the full calculation are shown by different lines. The left and right panels display the results without (Set 1$)$ and with (Set 2) $\kappa$ meson, respectively.

The largest cross section is obtained for the ${ }_{\Lambda}^{40} \mathrm{Cl}$ hypernucleus with $\Lambda$ in the $1 d_{5 / 2}$ state. The differential angular distribution for this case is analyzed in more detail in Fig. 4. From the comparison of the full and IA calculations we observe that the absorption of $\bar{p}$ and $\bar{\Lambda}$ has a quite significant effect: it reduces the cross section drastically, amounting at forward angles to about two orders of magnitude, and washes out the diffractive structures. Similar effects of the absorption are present also for the other $\Lambda$ states (not shown).

A deeper insight into the production mechanism is obtained by decomposing the total reaction amplitude into the different meson exchange parts. From the partial meson exchange contributions, shown in Fig. 4, it is remarkable that for Set 1 the kaon contribution is small and the spectrum is dominated by $K^{*}$, even at large angles, while on first sight one would expect the opposite. For example, for $\bar{p} A$ collisions at $p_{\text {lab }}=2 \mathrm{GeV} / \mathrm{c}$ the $\bar{\Lambda}$ produced at $\Theta_{\text {lab }}=30^{\circ}$ carries away the momentum transfer of $\sim 1 \mathrm{GeV} / \mathrm{c}$. This corresponds approximately to $\Theta=90^{\circ}$ in center-of-mass frame if translated into the $\bar{p} p \rightarrow \bar{\Lambda} \Lambda$ reaction in free space. Thus, we should expect that the kaon exchange should be a factor of five larger than the $K^{*}$ exchange. This surprising result can be understood by the fact that the momentum transfer to the $\bar{\Lambda}$ is provided by the nucleus as a whole while the hyperon is almost at rest. The exchange of a pseudoscalar meson is suppressed in this case since it proceeds through the lower of the proton and $\Lambda$ Dirac spinors which are suppressed by factors of the order of the baryon masses. In contrast, in the case of the free space $\bar{p} p \rightarrow \bar{\Lambda} \Lambda$ process at $\Theta=90^{\circ}$ the $\Lambda$ is produced with finite momentum and, thus, the upper and lower components of its Dirac spinor are of comparable magnitude which favors the pseudoscalar meson exchange. The situation is very different in the case of Set 2. Here, $\kappa$ plays the dominant role both for the free scattering $\bar{p} p \rightarrow \bar{\Lambda} \Lambda$ and for the hypernucleus production since scalar exchange is not suppressed in recoilless kinematics.

The robust signal of the $\kappa$ exchange is visible in the momentum dependence of the hypernucleus production cross section, as seen in Fig. 5. In calculations without $\kappa$ the cross section is dominated 

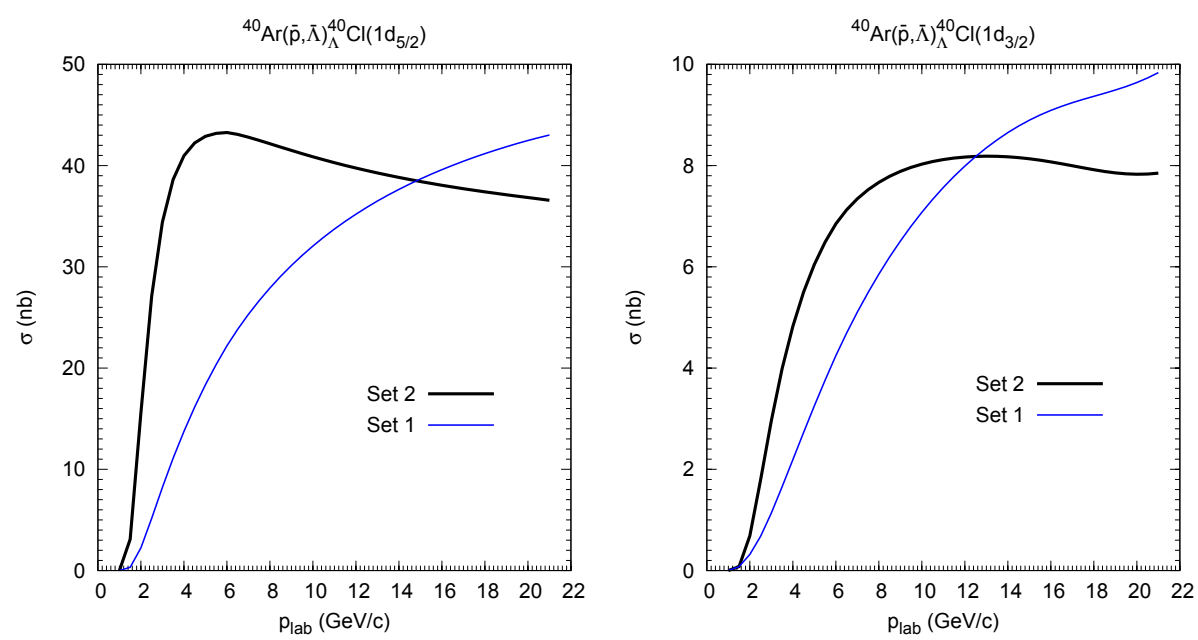

Figure 5. Beam momentum dependence of the ${ }_{\Lambda}^{40} \mathrm{Cl}$ d-state hypernucleus production cross section in $\bar{p}^{40} \mathrm{Ar}$ collisions. The thick and thin solid lines show, respectively, the results with (Set 2) and without (Set 1) $\kappa$ exchange.

by $K^{*}$ exchange which leads to a growing cross section with increasing beam energy. Using set 2 , the $\kappa$ meson dominates at moderate beam momenta $\sim 1.5 \ldots 3 \mathrm{GeV} / \mathrm{c}$. Its contributions are seen as a characteristic shoulder in $p_{\text {lab }}$-dependence of the hypernuclear production cross section and even as the appearance of the maximum for $1 d_{5 / 2} \Lambda$ state.

As we see from Fig. 6, the cross section of coherent hypernucleus production in different states is much larger when the $\kappa$ exchange is included. This is a pure quantum coherence effect since the angle-integrated $\bar{p} p \rightarrow \bar{\Lambda} \Lambda$ cross sections differ by $\sim 15 \%$ only at $p_{\text {lab }}=2 \mathrm{GeV} / \mathrm{c}$ (Fig. 2 ) while the hypernuclear production cross sections differ by almost one order of magnitude for Set 1 and Set 2 .

\section{Summary}

In summary, the coherent hypernucleus production in $\bar{p} A$ collisions was investigated. The production dynamics of the elementary and the in-medium annihilation amplitudes were described in a covariant meson exchange approach. Initial and final state interactions of the scattered baryons have been taken into account by eikonal theory. Baryon bound states were obtained by a RMF-model. The approach was applied to hypernuclear production in coherent reactions on the medium-heavy ${ }^{40} \mathrm{Ar}$ target nucleus in the momentum range $p_{\text {lab }} \sim 1.5 \ldots 20 \mathrm{GeV} / \mathrm{c}$. The total hypernucleus production cross sections populating a fixed quantum state generally grow with increasing beam momentum from several $\mathrm{nb}$ to a few of $10 \mathrm{nb}$ with a certain sensitivity on the $\Lambda$ bound state. The dynamics of the ${ }^{40} \operatorname{Ar}(\bar{p}, \bar{\Lambda})_{\Lambda}^{40} \mathrm{Cl}$ reaction on the $1 d_{3 / 2}$ valence shell proton favors the production of $\Lambda$ states with $j=\ell+1 / 2$ with the cross sections increasing with orbital angular momentum $\ell$.

The pseudoscalar $(K)$ exchange is strongly suppressed for the reactions replacing a bound proton by a bound $\Lambda$ hyperon. The production mechanism is governed, in fact, by the exchange of natural parity vector and scalar strange mesons. Including only $K^{*}$ exchange produces smooth and structureless cross sections increasing steadily with beam momentum for all possible bound $\Lambda$ states. However, if the exchange of the scalar $\pi K$ channel - described by the $\kappa$ meson - is taken into account we find 


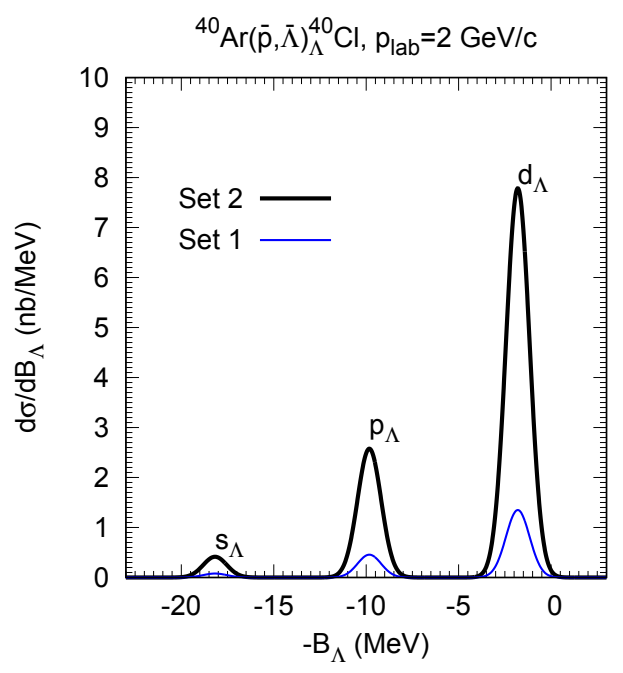

Figure 6. The $\Lambda$ binding energy spectrum of the ${ }_{\Lambda}^{40} \mathrm{Cl}$ hypernuclei coherently produced in $\bar{p}{ }^{40} \mathrm{Ar}$ collisions at $p_{\text {lab }}=2 \mathrm{GeV} / \mathrm{c}$. The smooth curves are obtained by multiplying the angle-integrated cross sections for the hypernucleus production in $1 s_{1 / 2}, 1 p_{3 / 2}$ and $1 d_{5 / 2}$ states by the Gaussians of a width FWHM=1.5 MeV which is a typical experimental energy resolution. Thick and thin solid lines correspond to the calculations with (Set 2) and without (Set 1) $\kappa$ exchange.

that at the beam momenta in the range of $p_{\text {lab }}=4 . . .6 \mathrm{GeV} / \mathrm{c}$ a rather sudden transition from increase to saturation occurs or, as in the case of the ${ }^{40} C l\left(1 d_{5 / 2}\right) \Lambda$ state, a maximum is emerging. These results strongly suggest that the coherent hypernuclear production in $\bar{p} A$ annihilation reactions could be a suitable tool to test in quite detail the dynamics of the production process, down to the possibility to identifying contributions from scalar $\pi K$ correlation as described by the $\kappa$ meson.

\section{Acknowledgments}

This work was supported by Deutsche Forschungsgemeinschaft (DFG) under Grant No. Le439/9-2.

\section{References}

[1] H. Bando, T. Motoba, J. Zofka, Int. J. Mod. Phys. A5, 4021 (1990)

[2] J. Cugnon, P. Deneye, J. Vandermeulen, Nucl. Phys. A513, 636 (1990)

[3] T. Gaitanos, A.B. Larionov, H. Lenske, U. Mosel, Nucl. Phys. A881, 240 (2012), 1111.5748

[4] T. Gaitanos, H. Lenske, Phys. Lett. B737, 256 (2014), 1408. 6051

[5] T. Gaitanos, C. Moustakidis, G.A. Lalazissis, H. Lenske, Nucl. Phys. A954, 308 (2016), 1602.08905

[6] H. Lenske, M. Dhar, T. Gaitanos, X. Cao, Progress in Particle and Nuclear Physics 98C, 119 (2018)

[7] A. Gal, E.V. Hungerford, D.J. Millener, Rev. Mod. Phys. 88, 035004 (2016), 1605.00557 
[8] R. Shyam, H. Lenske, U. Mosel, Nucl. Phys. A764, 313 (2006), nucl-th/0505043

[9] S. Bender, R. Shyam, H. Lenske, Nucl. Phys. A839, 51 (2010), 0910. 4868

[10] R. Shyam, H. Lenske, U. Mosel, Phys. Rev. C77, 052201 (2008), 0710. 4888

[11] K.A. Olive et al. (Particle Data Group), Chin. Phys. C38, 090001 (2014)

[12] R.G.E. Timmermans, T.A. Rijken, J.J. Deswart, Nucl. Phys. A479, 383C (1988)

[13] R.G.E. Timmermans, T.A. Rijken, J.J. de Swart, Phys. Rev. D45, 2288 (1992)

[14] J. Haidenbauer, U.G. Meissner, Phys. Rev. C72, 044005 (2005), nucl-th/0506019

[15] A.B. Larionov, H. Lenske, Nucl. Phys. A957, 450 (2017), 1609. 03343

[16] D. Garreta et al., Phys. Lett. 149B, 64 (1984)

[17] M.K. Cheoun, B.S. Han, I.T. Cheon, B.G. Yu, Phys. Rev. C54, 1811 (1996)

[18] K. Tsushima, A. Sibirtsev, A.W. Thomas, G.Q. Li, Phys. Rev. C59, 369 (1999), [Erratum: Phys. Rev.C61,029903(2000)], nucl-th/9801063

[19] B.S. Han, M.K. Cheoun, K.S. Kim, I.T. Cheon, Nucl. Phys. A691, 713 (2001), nucl-th/9912011

[20] A.B. Larionov, H. Lenske, Phys. Lett. B773, 470 (2017), 1709.04370

[21] R. Shyam, H. Lenske, Phys. Rev. D90, 014017 (2014), 1406.7071

[22] R. Shyam, H. Lenske, Phys. Rev. D93, 034016 (2016), 1509.04786

[23] J.J. de Swart, Rev. Mod. Phys. 35, 916 (1963), [Erratum: Rev. Mod. Phys.37,326(1965)]

[24] O. Dumbrajs, R. Koch, H. Pilkuhn, G.c. Oades, H. Behrens, J.j. De Swart, P. Kroll, Nucl. Phys. B216, 277 (1983)

[25] R. Machleidt, K. Holinde, C. Elster, Phys. Rept. 149, 1 (1987)

[26] G.A. Lalazissis, J. Konig, P. Ring, Phys. Rev. C55, 540 (1997), nucl-th/9607039

[27] C. Patrignani et al. (Particle Data Group), Chin. Phys. C40, 100001 (2016)

[28] R. Buettgen, K. Holinde, A. Mueller-Groeling, J. Speth, P. Wyborny, Nucl. Phys. A506, 586 (1990)

[29] A. Baldini et al., Landolt-Börnstein, V. 12 (Springer, Berlin, 1987)

[30] B. Jayet, M. Gailloud, P. Rosselet, V. Vuillemin, S. Vallet, M. Bogdanski, E. Jeannet, C.J. Campbell, J. Dawber, D.N. Edwards, Nuovo Cim. A45, 371 (1978)

[31] C.M. Keil, F. Hofmann, H. Lenske, Phys. Rev. C61, 064309 (2000), nucl-th/9911014

[32] N.K. Glendenning, D. Von-Eiff, M. Haft, H. Lenske, M.K. Weigel, Phys. Rev. C48, 889 (1993), nucl-th/9211012 